\title{
Apports lipidiques et prise de poids : aspects qualitatifs
}

Gérard AILHAUD

IBDC, Université de Nice Sophia-Antipolis, CNRS, 28 avenue de Valrose, 06100 Nice <ailhaud@unice.fr>
Abstract: The importance of dietary fat in human obesity remains a controversial issue as the prevalence of overweight and obesity has increased despite no dramatic change in the amount of ingested fats over the last decades. However qualitative changes, i.e. the fatty acid composition of fats, have been largely disregarded. In this review, we summarize experimental evidence which supports polyunsaturated fatty acids of the $\omega 6$ series as potent stimulators of both adipogenesis in vitro and adipose tissue development in vivo. Changes observed over the last decades in the fatty acid composition of dietary fats observed in breast milk and formula milk, i.e. increase in LA with lower or no change in LNA content, may be responsible at least in part of the dramatic rise in the prevalence of childhood overweight and obesity. Similar changes, which are observed in most consumed foods, can be traced to changes in human food habits and in the feeding pattern of breeding stock. These qualitative changes, acting in concert with a positive energy balance, should promote excessive adipose tissue development. Whether prevention of obesity appears critical to avoid difficult if not insurmountable health problems to solve in the future, the status of dietary lipids should be reconsidered from the very beginning of the food chain.

Key words: adipose tissue, linoleic acid, arachidonic acid, development, milk, foods

\section{Introduction}

Grâce à leurs acides gras de structure physicochimique très diverse, les lipides jouent de multiples rôles aux conséquences variées. Si les acides gras saturés, mono- et polyinsaturés peuvent subir un processus oxydatif, participer à la constitution des lipides complexes membranaires et s'accumuler sous la forme de triglycérides, seuls les acides gras polyinsaturés (AGPI) essentiels donnent naissance à de nombreux médiateurs lipidiques physiologiquement actifs. Parmi les AGPI, I'acide linoléique (C18:2 n-6 ; LA définissant la série $\omega 6$ ) donne naissance à l'acide dihomo- $\gamma$-linolénique (C18:3 n-6 ; DGLA) précurseur des prostaglandines (prostanoïdes) de la série 1 et des leucotriènes de la série 3 .

L'acide arachidonique (C20:4 n-6; ARA), issu du LA donne naissance à des médiateurs proinflammatoires (prostaglandines de la série 2 et leucotriènes de la série 4 ). L'acide $\alpha$-linolénique (C18:3 n-3 ; LNA définissant la série 103 ) donne naissance à l'acide eicosapentaénoïque (C20:5, $\mathrm{n}-3$; EPA) puis à l'acide docosahexaénoïque (C20:6 n-3; DHA). L'EPA peut donner naissance à des prostaglandines de la série 3 et des leucotriènes de la série 5 mais, plus encore, EPA comme DHA conduisent à des médiateurs antiinflammatoires tels que les resolvines et les protectines. Fait important, LA et LNA sont tous deux substrats de la delta-6-désaturase. Ainsi, in vivo, les flux métaboliques générés par LA et LNA dépendront initialement de leurs concentrations et donc du rapport LA/LNA au voisi- nage immédiat du site d'action de cet enzyme (figure 1).

La majorité des lipides de l'organisme trouve leur origine dans les aliments où ils représentent de $35 \%$ à $40 \%$ de l'énergie totale ingérée. Par l'intermédiaire des chylomicrons formés dans la muqueuse intestinale puis retrouvés dans la circulation, les acides gras se retrouvent, après quelques étapes enzymatiques, présents sous la forme de triglycérides dans le tissu adipeux, où ils pourront être restitués à la demande lors des besoins en énergie des muscles squelettique et cardiaque. Ainsi, même si une synthèse endogène d'acides gras saturés et mono-insaturés peut se produire à partir du glucose d'origine alimentaire, l'apport est majoritairement d'origine exogène.

De même, compte tenu des capacités limitées de l'enfant et de l'adulte à assurer la synthèse hépatique de l'ARA à partir de LA comme celle de I'EPA et du DHA à partir de LNA, ces acides gras polyinsaturés à longue chaîne proviennent très majoritairement de l'alimentation. Comme nous le verrons plus loin, ces AGPI en excès pourraient être à l'origine d'un problème majeur en induisant un développement excédentaire du tissu adipeux.

\section{Développement du tissu adipeux et lipides alimentaires : aspects qualitatifs}

Les conséquences d'une alimentation hypercalorique - le plus souvent hyperlipidique - sont bien connues chez les rongeurs et les primates, chez lesquels l'augmentation de la masse adipeuse se produit à la fois par une augmentation du nombre (hyperplasie) et du volume (hypertrophie) des adipocytes [1]. Chez l'Homme, les futurs obèses présentent dès la prime enfance une augmentation de ces deux paramètres plus rapidement que les individus normopondéraux et le phénomène persiste tout au long de la vie adulte [2]. Le développement du tissu adipeux, caractérisé par la formation d'adipocytes, se produit à partir de cellules précurseurs (préadipocytes) au cours du troisième trimestre de la grossesse puis après la naissance [3] mais reste possible à tout âge, des préadipocytes étant retrouvés dans le tissu adipeux souscutané chez les octogénaires des deux sexes [4]. Contrairement aux adipocytes, les cellules précurseurs peuvent se diviser. Cet évènement prolifératif est pondéralement "silencieux ", ces cellules étant 30 à 50 fois moins volumineuses que les adipocytes. La prolifération cellulaire dans le tissu adipeux blanc humain chez l'adulte apparaît comme un processus lent (demi-vie de 240 à 425 jours) [5]. Une fois formés, le renouvellement et/ou la disparition dans des conditions physiologiques des adipocytes est peu important et concerne, consécutivement à une perte de poids, les macrophages qui sont très présents en nombre important dans le tissu adipeux des individus obèses [1, 6-8]. En d'autres termes, la formation des adipocytes est un processus quasi-irréversible, susceptible de se poursuivre à l'âge adulte. La prévention reste donc déterminante pour contrôler le développement du tissu adipeux 


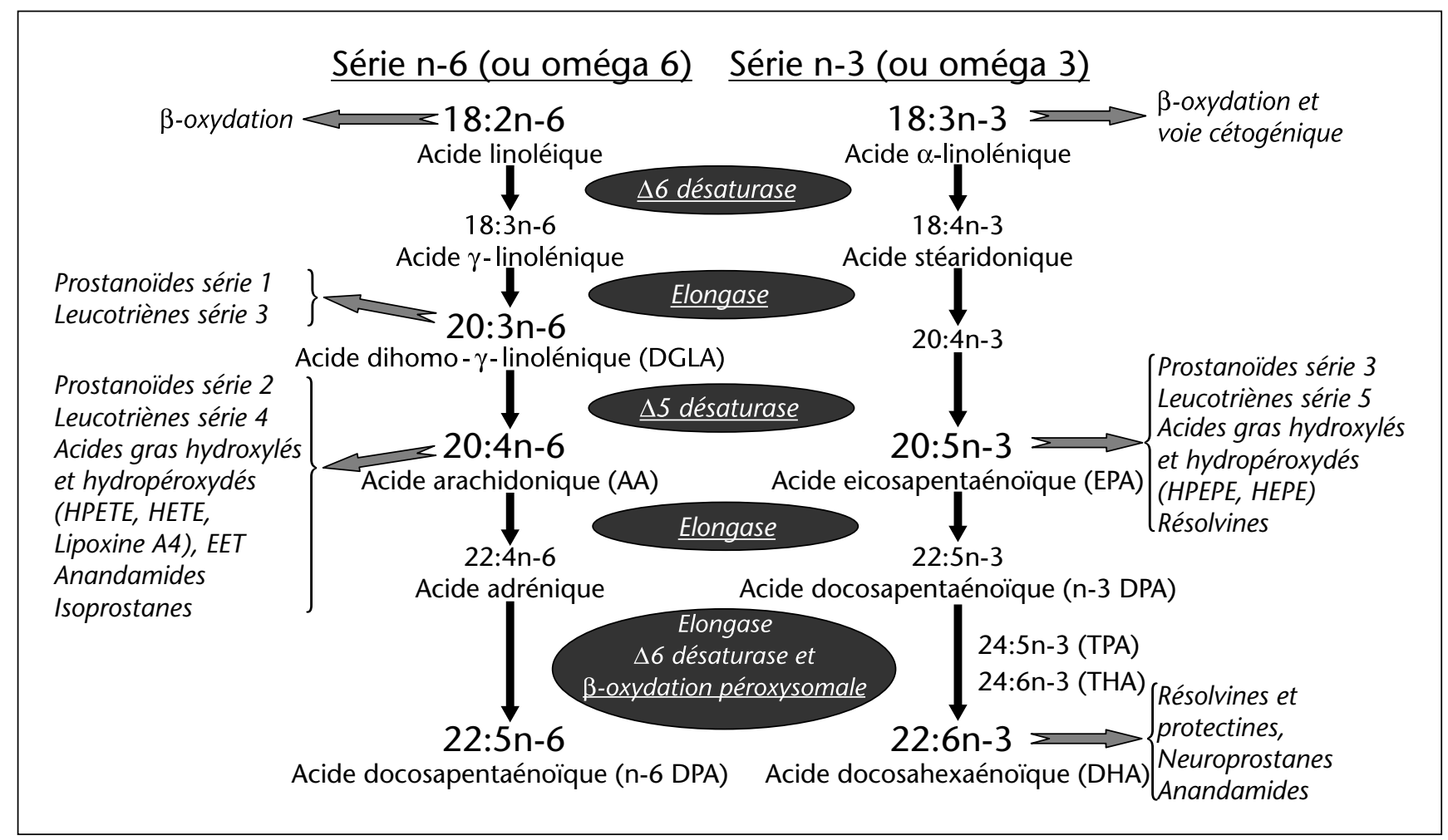

Figure 1. Médiateurs lipidiques générés à partir des acides linoléique et $\alpha$-linolénique. La nature des nombreux médiateurs lipidiques générés à partir des acides gras polyinsaturés des séries $\omega 6$ et $\omega 3$ dépend du type cellulaire considéré et de la chronologie des évènements cellulaires. Ainsi, la production par les leucocytes de métabolites pro-inflammatoires à partir d'ARA (prostaglandines de la série 2 par exemple) initie la production de métabolites anti-inflammatoires à partir d'EPA et de DHA (resolvines, protectines) qui arrêtent l'ensemble du processus. Les préadipocytes produisent $P G I_{2}$ (prostacycline), $P G E_{2}$ et des traces de PGF $F_{2 \alpha}$; la production de métabolites issus de EPA et de DHA n'a pas été démontrée.

au cours duquel les facteurs nutritionnels, et en particulier les lipides, vont jouer un rôle très important. En effet, des données récentes montrent en France, entre 1960 et 2000, une augmentation de $40 \%$ de la consommation de lipides [9]. Plus encore, les changements intervenus dans la nature des acides gras des lipides alimentaires sont restés ignorés mais posent problème [10-13].

On sait qu'une obésité précoce est prédictive d'une obésité ultérieure [14]. Ainsi, l'index de masse corporelle d'enfants de 8 ans est positivement associé à celui préalablement déterminé à l'âge de 2 ans [15]. La connaissance des évènements cellulaires et moléculaires qui gouvernent la formation du tissu adipeux est de ce fait déterminante.

\section{Adipogénèse in vitro et in vivo : aspects qualitatifs}

La caractérisation des facteurs nutritionnels et hormonaux impliqués dans la différenciation adipocytaire (adipogénèse) montre que les facteurs hormonaux requis sont peu nombreux in vitro et que leurs taux circulants ou locaux sont associés in vivo soit à l'état nutritionnel [insuline, facteur insulino-mimétique (IGF-1)] soit à l'activation de l'axe hypothalamo-hypophysosurrénalien et/ou à une synthèse in situ (glucocorticoïdes). Les acides gras naturels se révèlent comme de véritables hormones adipogéniques qui sont actives sur les préadipocytes en lignée établie comme sur les préadipocytes isolés à partir de tissu adipeux de rat et d'homme [1]. Ces observations établissent un lien direct entre lipides alimentaires, flux d'acides gras pénétrant le tissu adipeux, et formation d'adipocytes. Un lien moléculaire a pu être établi grâce au clonage de récepteurs nucléaires de la famille des peroxisome proliferator-activated receptors (PPAR) [16] qui se comportent comme des détecteurs à la fois des acides gras naturels et de certains métabolites de l'ARA [17]. Fait important, tous les acides gras n'apparaissent pas équipotents pour stimuler l'adipogénèse. Ainsi I'ARA (série $\omega 6$ ), le plus représenté dans I'alimentation, est 3 fois plus adipogénique que son équivalent de la série $\omega 3$ (présent naturellement à l'état de traces) et plus puissant que les acides gras saturés (palmitate), mono-insaturés (oléate, palmitoléate) et EPA, le $\mathrm{DHA}$ se révélant même anti-adipogénique $[18,19]$. L'effet adipogénique de l'ARA passe par sa capacité à donner naissance dans les préadipocytes à de la prostacycline. Après sa sécrétion, la liaison de cette prostaglandine à son récepteur de surface IP-R, qui est couplé positivement à l'adénylate cyclase, entraîne la production d'AMP cyclique et l'activation de la voie dépendante de la protéine kinase A [20, 21]. Cette dernière stimule alors l'adipogénèse via l'expression des CCAT/enhancer binding proteins (C/EBP) $\beta$ et $\delta$ [22]. Ces deux facteurs transcriptionnels modulent positivement à leur tour l'expression de PPAR $\gamma$ qui gouverne finalement la différenciation terminale et conduit à la formation d'adipocytes [1].

In vivo comme in vitro, les acides gras polyinsaturés ne sont pas équipotents pour promouvoir le développement du tissu adipeux. Chez le rat, un régime enrichi en huile de poisson riche en EPA et DHA n'entraîne pas d'obésité [23]. De même, un régime hyperlipidique riche en LNA précurseur de I'EPA et du DHA empêche le développement excessif du tissu adipeux [24]. Àl'inverse, chez des souriceaux nés puis allaités par des mères nourries pendant quelques générations avec un régime riche en $L A$ précurseur de l'ARA, on observe après sevrage une augmentation de $40 \%$ de leur poids corporel par rapport aux animaux nourris avec un régime standard. Cette différence est maintenue à l'âge adulte et l'effet adipogénique du LA 
est contrecarré, dans des conditions isoénergétiques et isolipidiques, par un apport en LNA. L'inactivation du gène codant pour le récepteur IP-R abolit l'effet stimulateur du LA sur la masse grasse des souriceaux, démontrant que cet effet passe via ARA par une production de prostacycline [18]. Des résultats obtenus chez le souriceau [25] et chez le raton [26] sont également en faveur d'un rôle adipogénique joué par LA et soulignent l'importance du rapport LA/LNA dans le développement précoce du tissu adipeux. Une situation analogue est observée chez des enfants nés à terme et nourris avec $16 \% \mathrm{LA}$, chez lesquels une supplémentation pendant 4 mois avec 3,2 \% LNA (rapport LA/LNA de 4,8) entraîne une augmentation du DHA et une diminution de I'ARA des lipides circulants qui s'accompagnent d'une prise de poids plus faible que celle observée par une supplémentation avec 0,4\% LNA (rapport LA/LNA de 44) [27]. L'exemple le plus spectaculaire du rôle adipogénique joué par LA a été démontré voici 40 ans chez l'homme adulte. Des personnes du troisième âge vivant en institution avaient été maintenues dans des conditions nutritionnelles où la seule substitution avait été de remplacer en grande partie les acides gras saturés par de l'acide linoléique pendant 5 ans. Dans ces conditions isocaloriques et isolipidiques, le poids corporel moyen du groupe expérimental avait augmenté alors que celui du groupe contrôle avait diminué (différence de $5 \% ; 400$ sujets par groupe). Le gain de poids était corrélé positivement avec la proportion de LA présent dans les acides gras totaux qui avait été déterminée à partir de biopsies de tissu adipeux sous-cutané [28]. De manière spectaculaire, alors que 3 à 5 ans s'étaient révélés nécessaires chez ces personnes âgées pour que la proportion de LA des lipides du tissu adipeux sous-cutané s'équilibre avec celle présente dans les lipides alimentaires, 6 semaines sont suffisantes dans le cas d'enfants nés à terme, précisément au moment même où le développement de la masse adipeuse augmente parallèlement à celle des autres organes [29]. On voit donc que le nouveau-né répond très rapidement aux stimuli nutritionnels de nature lipidique.

\section{Évolution des apports alimentaires en acides gras polyinsaturés}

Compte tenu de l'augmentation de la prévalence du surpoids et de l'obésité depuis quelques décennies, il est important de déterminer si des changements importants ont été parallèlement observés dans les apports alimentaires en AGPI des séries $\omega 3$ et $\omega 6$ pendant cette période, d'autant plus que le traitement de l'hypercholestérolémie avait conduit à recommander les acides gras polyinsaturés au détriment des acides gras saturés. Les résultats montrent que la seule proportion d'AGPI de la série $\omega 6$ a considérablement augmentée dans le lait maternel, les laits maternisés et la majorité des aliments consommés par l'enfant et l'adulte [9]. Concernant le lait maternel, entre 1950 et 1995, la proportion du LA est passée aux USA de $6 \%$ à $18 \%$ alors que celle de LNA restait stable à $1 \%$. Ces proportions sont similaires à celles trouvées dans les lipides totaux provenant de biopsies de tissu adipeux sous-cutané de femmes américaines adultes et que l'on sait être un reflet fidèle de leurs ingesta, avant et pendant leur grossesse, tout comme au cours de l'allaitement. Par ailleurs, correctement évalués à partir de 1980, le rapport ARA/DHA du lait mature de femmes européennes comme américaines a augmenté, avec des valeurs supérieures de $50 \%$ pour les USA. Dans le cas des laits infantiles, dont la composition se veut un reflet adéquat de celle du lait maternel qui est en fait variable, le rapport LA/LNA se situe depuis 1995 autour de valeurs proches de celles recommandées (entre 8,5 et 21,7 ) avec toutefois un taux toujours élevé de LA ( 9 à $26 \%$ des acides gras totaux) [9]. Ce taux reste important au regard des recommandations faites par les comités d'experts mais surtout au regard des besoins réels en LA qui apparaissent très surestimés [30]. Notons à ce propos le cas de bébés de 6 à 11 mois, dont l'index relatif d'adiposité supérieur au $95^{\mathrm{e}}$ percentile de la courbe de référence a doublé aux USA entre les années 1976-1980 et 1988-1994 [31], et chez lesquels une augmentation des ingesta et/ou une diminution des dépenses énergétiques peuvent difficilement être avancées à cet âge pour expliquer un tel phénomène. Celui-ci paraît au contraire de nature qualitative et en accord avec l'augmentation des rapports LA/LNA et ARA/DHA observée parallèlement. Lorsque les bébés sont nourris au lait maternel, à long terme, le contenu en ARA et DHA est proche de celui déterminé dans l'alimentation de la mère mais ne reflète pas le contenu en LA et LNA consommés. Une consommation accrue d'ARA par la mère n'a pas d'effet sur la proportion d'ARA dans le lait maternel mais par contre entraîne une réduction de EPA et DHA, conduisant ainsi à une augmentation des rapports ARA/DHA et ARA/EPA + DHA. Dans tous les cas, les niveaux circulants d'ARA et/ou la valeur du rapport ARA/DHA(+EPA) augmentent d'autant plus dans les lipides circulants chez le bébé que I'apport en AGPI de la série $\omega 3$ est faible, ce qui correspond précisément aux conditions nutritionnelles qui prévalent dans la majorité des pays industrialisés. Dans le cas des laits infantiles supplémentés en ARA et DHA (rapport ARA/DHA compris entre 1,5 et 2 et proche du lait maternel), les proportions de LA et LNA ont peu d'effet sur les niveaux circulants d'ARA et des AGPI de la série 03 qui sont en fait déterminés par leur proportion dans les laits supplémentés [9].

II est intéressant de constater que la situation nutritionnelle existant dans la prime enfance se prolonge chez l'enfant et l'adulte. A partir de I'analyse des acides gras du tissu adipeux d'adultes (biomarqueurs fiables des ingesta) on constate, pendant la période s'étalant de 1960 à 2000, un enrichissement en LA et un appauvrissement en LNA, le rapport LA/LNA passant de 17 à 21,6 aux USA et de 6,8 à 13,6 en Europe. En France, au cours de la même période, à partir de données de production, on a pu estimer que les consommations de LA et d'ARA se sont accrues respectivement de 250 et $230 \%$ alors que celle de LNA a diminué de $40 \%$, avec un quadruplement du rapport LA/LNA, le tout associé à une consommation en LA 2 fois supérieure et celle de LNA 2 fois inférieure aux apports nutritionnels conseillés [9].

\section{Conclusion}

II est admis que l'augmentation de la prévalence du surpoids et de l'obésité, liée à une balance énergétique positive, serait principalement due à une diminution des dépenses énergétiques sans augmentation des apports caloriques, dont on sait qu'ils sont le plus souvent apportés par une consommation importante de matières grasses. En France, la consommation de lipides est passée entre 1960 et 2000 de $75 \mathrm{~g} / \mathrm{j}$ à $104 \mathrm{~g} / \mathrm{j}$. Dans le même temps, des changements importants sont intervenus, essentiellement dus à une consommation accrue d'huiles végétales et aux modifications de l'alimentation animale, qui se sont globalement traduits par un enrichissement substantiel en acides gras polyinsaturés de la série $\omega 6$ dans notre alimentation. La combinaison de deux évènements, quantitatif et qualitatif, aurait donc contribué à l'augmentation de la prévalence du surpoids et de l'obésité au cours des dernières décennies.

\section{RÉFÉRENCES}

1. AILHAUD G, HAUNER H. Development of white adipose tissue. In : Bray AG, Bouchard C, eds. Handbook of Obesity: Etiology and Pathophysiology, Second Edition. New York : Marcel Dekker, Inc., 2004 : 481-514 ; (chapter 17).

2. KNITTLE JL, TIMMERS K, GINSBERG-FELLNER F, BROWN RE, KATZ DP. The growth of adipose tissue in children and adolescents. Crosssectional and longitudinal studies of adipose cell number and size. / Clin Invest $1979 ; 63: 239-46$.

3. POISSONNET CM, BURDI AR, BOOKSTEIN FL. Growth and development of human adipose tissue during early gestation. Early Hum Dev $1983 ; 8: 1-11$. 
4. HAUNER H, ENTENMANN M, WABITSCH M, ET AL. Promoting effect of glucocorticoids on the differentiation of human adipocyte precursor cells cultured in a chemically defined serum. J Clin Invest 1989 ; 84 : 1663-70.

5. STRAWFORD A, ANTELO F, CHRISTIANSEN M, KELLERSTEIN MK. Adipose tissue triglyceride turnover, de novo lipogenesis and cell proliferation in humans measured with ${ }^{2} \mathrm{H}_{2} \mathrm{O} . \mathrm{Am} /$ Physiol Endocrinol Metab 2003; 286: E577. E588.

6. WEISBERG SP, MCCANN D, DESAI M, ROSENBAUM M, LEIBEL RL, FERRANTE JR. AW. Obesity is associated with macrophage accumulation in adipose tissue. / Clin Invest $2003 ; 112$ : 1796-808.

7. CINTI S, MITCHELL G, BARBATELLI G, ET AL. Adipocyte death defines macrophages localization and function in adipose tissue of obese mice and humans. J Lipid Res 2005 ; 46 : 2347 55.

8. CANCELLO R, HENEGAR C, VIGUERI N, ET AL Reduction of macrophage infiltration and chemoattractant gene expression changes in white adipose tissue of morbidly obese subjects after surgery-induced weight loss. Diabetes 2005 ; $54: 2277-86$

9. AILHAUD G, MASSIÉRA F, WEILL P, LEGRAND P, ALESSANDRI JM, GUESNET P. Temporal changes in dietary fats : role of $n-6$ polyunsaturated fatty acids in excessive adipose tissue development and relationship to obesity. Prog Lipid Res 2006; 45 : 203-36.

10. SIMOPOULOS AP. The importance of the ratio of omega-6/omega-3 essential fatty acids. Biomed Pharmacother $2002 ; 56: 365-79$.

11. HIBBELN JR, NIEMEN LR, BLASBAG TL, ET AL. Healthy intakes of $n-3$ and $n-6$ fatty acids: estimations considering worldwide diversity. Am J Clin Nutr 2006 ; 83 : 1483S-1493S.
12. OKUYAMA H, ICHIKAWA Y, SUN Y, ET AL. Cancers common in the USA are stimulated by omega 6 fatty acids and large amounts of animal fats, but suppressed by omega 3 fatty acids and cholesterol. World Rev Nutr Diet 2007 ; 96 : 143-9.

13. DUBNOV G, BERRY EM. Omega- 6 fatty acids and coronary artery disease: the pros and cons. Curr Atheroscler Rep 2004 ; 6 : 441-4.

14. BAIRD B, FISHER D, LUCAS P, ET AL. Being big or growing fast : systematic review of size and growth in infancy and later obesity. Br Med / 2005 ; (October).

15. SKINNER JD, BOUNDS W, CARRUTH BR, MORRIS M, ZIEGLER P. Predictors of children's body mass index : a longitudinal study of diet and growth in children aged 2-8 y. Int J Obes Relat Metab Disord 2004 ; 28 : 476-82.

16. HIHI AK, MICHALIK L, WAHLI W. PPARs : transcriptional effectors of fatty acids and their derivatives. Cell Mol Life Sci 2002 ; 59 : 790-8.

17. AMRI EZ, AILHAUD G, GRIMALDI P. Fatty acids as signal transducing molecules : involvement in the differentiation of preadipose to adipose cells. J Lipid Res 1994 ; 35 : 930-7.

18. MASSIERA F, SAINT-MARC $P$, SEYDOUX J, ET AL. Arachidonic acid and prostacyclin signaling promote adipose tissue development: a human health concern? / Lipid Res 2003 ; 44 : 271-9.

19. KIM HK, DELLA-FERRA MA, LIN J, ET AL. Docosahexaenoic acid inhibits adipocyte differentiation and induces apoptosis in 3T3-L1 preadipocytes. J Nutr 2006 ; 136 : 2965-9.

20. GAILLARD D, NEGREL R, LAGARDE M, AILHAUD G. Requirement and role of arachidonic acid in the differentiation of pre-adipose cells. Biochem / $1989 ; 257$ : 389-97.

21. VASSAUX G, GAILLARD D, AILHAUD G, NÉGREL R. Prostacyclin is a specific effector of adipose cell differentiation. Its dual role as a CAMP- and $\mathrm{Ca}(2+)$-elevating agent. J Biol Chem 1992 ; $267: 11092-7$.
22. BELMONTE N, PHILLIPS BW, MASSIERA F, ETAL. Activation of extracellular signalregulated kinases and CREB/ATF-1 mediate the expression of CCAAT/enhancer binding proteins beta and -delta in preadipocytes. Mol Endocrinol $2001 ; 15$ : 2037-49.

23. PARRISH CC, PATHY D, ANGEL A. Dietary fish oils limits adipose tissue hypertrophy in rats. Metabolism $1990 ; 39$ : 217-9.

24. RACLOT T, GROCOLAS R, LANGIN D, FERRE P. Site-specific regulation of gene expression by n-3 polyunsaturated fatty acids in rat white adipose tissues. / Lipid Res 1997 ; 38 : 1963-72.

25. JAVADI M, EVERTS $H$, HOVENIRE R, ET AL. The effect of six different $\mathrm{C} 18$ fatty acids on body fat and energy metabolism in mice. $\mathrm{Br} / \mathrm{Nutr}$ $2002 ; 92: 391-9$.

26. KOROTKOVA M, GABRIELSSON B, LONN M, HANSON LA, STRANDVIK B. Leptin levels in rat offspring are modified by the ratio of linoleic to alpha-linolenic acid in maternal diet. / Lipid Res $2002 ; 43: 1743-9$.

27. JENSEN CL, PRAGER TC, FRALEY IK, CHEN H, ANDERSON RE, HEIRD WC. Effect of dietary linoleic/alpha-linolenic acid ratio on growth and visual function of term infants. J Pediatr $1997 ; 131$ : 200-9.

28. DAYTON S, HASHIMOTO S, DIXON W, PEARCE ML. Composition of lipids in human serum and adipose tissue during prolonged feeding of a diet high in unsaturated fat. / Lipid Res 1986 ; $7:$ 103-11.

29. SWEENEY MJ, ETTELDORF JN, THROOP LJ, TIMMA DL. Diet and fatty acid distribution in subcutaneous fat and in the cholesteroltriglyceride fraction of serum of yound infants. J Clin Invest $1963 ; 42$ : 1-9.

30. CUNNANE SC. Problems with essential fatty acids : time for a new paradigm? Prog Lipid Res $2003 ; 42$ : 544-68.

31. OGDEN CL, TROIANO RP, BRIEFEL RR, KUCZMARSKI RJ, FLEGAL KM, JOHNSON CL. Prevalence of overweight among preschool children in the United States, 1971 through 1994. Pediatrics 1994 ; 99 : E1. 